\begin{tabular}{|c|l|}
\hline Title & $\begin{array}{l}\text { Cross Sectional Evolution and Its Mechanism during Selective Molecular Beam Epitaxy Growth of GaA s Quantum } \\
\text { Wires on (111)B Substrates }\end{array}$ \\
\hline Author(s) & Tamai, I sao; Sato, Taketomo; Hasegawa, Hideki \\
\hline Citation & $\begin{array}{l}\text { Japanese Journal of A pplied Physics. Pt. 1, Regular papers, brief communications \& review papers, 44(4B), 2652-2656 } \\
\text { https://doi.org/10.1143/JAP.44.2652 }\end{array}$ \\
\hline Issue Date & 2005-04 \\
\hline Doc URL & http://hdl.handle.net/2115/33095 \\
\hline Rights & Copyright $\odot$ 2005 The Japan Society of A pplied Physics \\
\hline Type & article (author version) \\
\hline File Information & TSA TO18.PDF \\
\hline
\end{tabular}

Instructions for use 


\title{
Cross-Sectional Evolution and Its Mechanism during Selective Molecular Beam Epitaxy Growth of GaAs Quantum Wires on (111)B Substrates
}

\author{
Isao Tamai*, Taketomo Sato and Hideki Hasegawa \\ Graduate School of Graduate School of Information Science and Technology and \\ Research Center for Integrated Quantum Electronics, Hokkaido University, \\ North-13, West-8, Sapporo 060-8628, Japan \\ *Corresponding author. E-mail address: tamai@rciqe.hokudai.ac.jp
}

The mechanism of the cross-sectional evolution during the selective growth of GaAs quantum wires (QWRs) on (111)B-patterned substrates was studied in detail both experimentally and theoretically. For this purpose, growth experiments were carried out on $<-1-12>$-oriented wires by systematically changing growth conditions and pattern sizes. A detailed investigation on cross sections of wires has shown that the lateral wire width is determined by facet boundaries (FBs) within AlGaAs layers separating growth regions on top facets from those on side facets of mesa structures. FBs were found to be planar or curved, depending on initial pattern sizes and growth conditions. Computer simulation based on a phenomenological growth model was attempted, taking account of the facet-angle-dependent lifetime of adatoms. The simulation well reproduced the experimentally observed growth features including the evolution of FBs, indicating that the cross sections of wires grown on (111)B-patterned substrates are kinetically controlled by the pattern sizes and growth conditions.

KEYWORDS: selective growth, molecular beam epitaxy (MBE), (111)B-patterned substrates, growth mechanism, GaAs, AlGaAs

\section{Introduction}

For the realization of next-generation large-scale integrated circuits (LSIs) based on quantum nanodevices, high-density arrays and networks of quantum dots (QDs) and quantum wires (QWRs) are strongly demanded. Among various nanostructure formation techniques, selective growth by molecular beam epitaxy (MBE) or metal-organic vapor phase epitaxy (MOVPE) using prepatterned or masked substrates [1-5] is one of the most promising techniques for the fabrication of quantum nanostructures with high controllabilities of position and size below those achievable by standard lithography techniques.

As a new and realistic approach for the large-scale integration of quantum devices on nanostructure networks, our group has recently proposed a hexagonal binary decision diagram (BDD) quantum circuit approach [6,7] Here, path-switching BDD node devices having three branches are formed on hexagonal QWR networks to achieve a maximum packing density. We have also demonstrated the feasibility of growing hexagonal AlGaAs/GaAs QWR networks on (001) [8,9] and (111)B GaAs [10] substrates by selective MBE. In particular, the three-fold symmetry of the latter (111)B substrate is inherently favorable for the formation of hexagonal QWR networks with node devices having three branches. This is because the wire cross sections meeting at the hexagon node point can be made the same so that the electrical connection of wires at the node point will become much easier than in the case of growth on (001) substrates where wire cross sections are very different, depending on the wire direction. However, selective growth on nonplanar (111)B substrates is complex due to growth kinetics involving various high-index facets. Thus, proper understanding of the growth mode and 
mechanism is indispensable for the precise control of the size and position of selectively grown QWRs.

The purpose of this study is to investigate, both experimentally and theoretically, the evolution of wire cross sections and its mechanism during the selective growth of $<-1-12>-$-oriented QWRs on (111)B-patterned substrates. For this purpose, growth experiments were carried out by systematically changing growth conditions and pattern sizes. A detailed investigation on cross sections of wires has revealed that the lateral wire width is determined by facet boundaries (FBs) within AlGaAs layers separating growth regions between the neighboring facets of mesa structures. Computer simulation based on a phenomenological growth model has well reproduced the experimentally observed growth features including the evolution of FBs, indicating that the wire cross sections are kinetically controlled by growth conditions and initial mesa shapes.

\section{Experiments of Selective Growth on (111)B Substrates \\ 2.1 Method of growth}

The sequence for selective MBE growth used in this study is schematically shown in

Fig. 1. As a template for selective MBE growth, arrays of $<-1-12>$-oriented mesa stripes shown in Fig. 1(a) are formed on semi-insulating (111)B GaAs substrates. Patterns are prepared by standard electron beam (EB) lithography and $\mathrm{HBr}$-based etching using $\mathrm{H}_{2} \mathrm{O}$ : $\mathrm{HBr}$ : saturated brome water $(\mathrm{SBW})=100: 20: 8$.

Before loading substrates into an MBE chamber, a surface treatment is applied using an organic solution and light chemical etching in the atmosphere. Then, thermal cleaning at a substrate temperature of $640^{\circ} \mathrm{C}$ under an arsenic pressure is applied in the MBE chamber prior to growth. A typical material supply sequence is shown in Fig. 1(b). First, a GaAs buffer layer is grown on a patterned substrate as shown in Fig. 1(c) in order to prepare a growth template for subsequent selective growth. In our previous study [10], buffer growth led to a GaAs mesa structure defined by top (111)B and side (5-12) facets. Then, an $\mathrm{Al}_{0.3} \mathrm{Ga}_{0.7} \mathrm{As} / \mathrm{GaAs} / \mathrm{Al}_{0.3} \mathrm{Ga}_{0.7} \mathrm{As}$ sandwich layer is grown on the buffer template, and this leads to the formation of embedded GaAs QWRs on the top (111)B facets of the AlGaAs mesa structure with a reduced lateral wire width as shown in Fig. 1(d).

In this study, systematical growth experiments were performed on the above-mentioned GaAs buffer mesa in order to clarify the basic growth behavior on (111)B-patterned substrates. For this, buffer mesa patterns with various values of height, $h_{0}$, and width, $W_{0}$, as defined in Fig. 1(c), were prepared. Various growth data related to cross-sectional structures were collected in order to use them later in the theoretical calculation based on a phenomenological model.

As basic growth conditions, the V/III ratio and the growth temperature, $T_{\text {sub, }}$, were set to be 10 and $700^{\circ} \mathrm{C}$, respectively, for all experiments. The growth rate of AlGaAs was set to be $1000 \mathrm{~nm} / \mathrm{h}$ for planar growth.

\subsection{Wire cross section and facet boundary}

The growth experiments confirmed the selective growth of quantum wire arrays, whose wire cross section is shown in Fig. 1(d). To clarify how the wire width and position are determined in the case of $<-1-12>$-oriented QWRs, wire growth was repeated on the same GaAs buffer mesa structures having various values of initial width, $W_{0}$, and height, $h_{0}$, as defined in Fig. 1(c).

Figure 2(a) shows the cross-sectional SEM image of a sample obtained after repeated growth on a large buffer mesa structure with $W_{0}=2600 \mathrm{~nm}$ and $h_{0}=750 \mathrm{~nm}$. In such a case of 
growth on the large mesa template, it is clearly seen that the boundaries separating the growth regions between the top and side facets form a plane within the AlGaAs layer, which we call a facet boundary plane (FBP). This maintains the facet boundary angle, $\theta_{\mathrm{b}}$, defined as the angle of a facet boundary with respect to the base (111)B plane with a constant value of $69^{\circ}$ throughout the growth. The lateral wire width of the QWR, $W$, is then determined by the top mesa width marked out by a set of two FBPs.

However, it was found that the situation becomes more complex when the height of the buffer mesa decreases. Examples are shown in Figs. 2(b) and 2(c) for cases of $h_{0}=250 \mathrm{~nm}$ and $W_{0}=1220 \mathrm{~nm}$, and $h_{0}=250 \mathrm{~nm}$ and $W_{0}=620 \mathrm{~nm}$, respectively. Surprisingly, it was found in the case shown in Fig. 2(b) that the facet boundary (FB) was no longer a plane, but was curved. Namely, the facet boundary angles changed with growth time. Moreover, the facet boundary angle remained at $69^{\circ}$ for the growth of the first four or five layers, and then it increased to more than $90^{\circ}$. In this case, the wire width initially decreased with time, and then started to increase after reaching a minimum value. On the other hand, when the initial width was smaller as in the case shown in Fig. 2(c) for the same value of $h_{0}=250 \mathrm{~nm}$, the boundary angle also remained constant within the growth time considered, but it decreased to $65^{\circ}$, resulting in a marked decrease in wire width with growth time.

\subsection{Variation in wire width with growth time}

The observed change in wire width, $W$, with growth time is plotted in Fig. 3. All data of wire width, $W$, were normalized by $W_{0}$. The solid lines show the results of computer simulation which will be explained later.

The wire width of the sample grown on a larger mesa having larger $W_{0}$ and larger $h_{0}$, linearly decreased with growth time due to the constant angle of $\theta_{\mathrm{b}}$ throughout the entire growth. On the other hand, as for the sample grown on a shallow mesa, wire width decreased for the first 30 or $40 \mathrm{~min}$ of growth, but later, gradually increased with growth time. This behavior is a consequence of the change in facet boundary angle with growth time, as seen in Fig. 2(b).

To further acquire quantitative data for the evolution of wire cross sections, vertical growth rates on different facets were measured by changing the growth temperature. In this experiment, a large mesa pattern having $W_{0}=2600 \mathrm{~nm}$ and $h_{0}=750 \mathrm{~nm}$ was used in order to avoid complex effects seen in the growth on the shallow mesa substrate. The measured growth rate on the top facet, $t_{\text {top }}$, and that on the side facet, $t_{\text {side, }}$ are plotted in Fig. 4 as functions of growth temperature, $T_{\text {sub }}$. Here, the $t_{\text {top }}$ and $t_{\text {side }}$ values are normalized by the growth rate on the planar (111)B substrate, $t_{\text {planar. }}$ As the temperature increased, the growth thickness on the top facet increased, whereas that on the side facet decreased. This strongly suggests that there exist large differences in migration and atom incorporation rates between the top and side facets.

\subsection{Growth experiment on one-sided mesa}

To understand the complex behavior of wire growth as presented in the previous section, growth experiments on a more basic structure of a one-sided mesa step were carried out. Figure 5 shows the cross-sectional SEM image of a sample obtained after the repeated growth of AlGaAs $(100 \mathrm{~nm}) / \mathrm{GaAs}(10 \mathrm{~nm})$, where GaAs was used as a marker. The initial GaAs buffer mesa had a side (5-12) facet with a height of $h_{0}=250 \mathrm{~nm}$ whose value is the same as that used for wire growth experiments shown in Figs. 2(b) and 2(c).

The lines corresponding to GaAs markers in Fig. 5 show complex spatial patterns similar to those seen in Figs. 2(b) and 2(c). By closely observing Fig. 5, it was found that the 
angle of the side facet of the mesa, $\theta$, decreased with growth time, as shown in Fig. 5. This indicates that the accumulation of a material due to diffusion and incorporation at the bottom next to the initial buffer mesa causes a continuous change in side facet angle.

\section{Computer Simulation of Selective Growth}

\subsection{Basic equations}

The complex behavior of the evolution of cross-sectional structures observed in the experiment is difficult to describe using a simple analytical relation, as we previously observed for growth on a (001) substrate [11]. As a means of quantitative theoretical description of the complex behavior of the selective MBE growth, an attempt was made here to reproduce the growth profile of the $<-1-12>$-oriented mesa structure on the (111)B substrate by computer simulation using a phenomenological growth model, in which the growth process is described by macroscopic parameters such as diffusion constant and lifetime. Such a calculation has been previously carried out for the $<-1-10>$-oriented QWR on the (001) substrate [12]. In this modeling, the surface density of group III adatoms, $n\left(x, t_{g}\right)$, at the lateral position, $x$, and the growth time, $t_{g}$, is assumed to satisfy the following phenomenological equation:

$$
\begin{aligned}
& \frac{\mathrm{d} n\left(x, t_{g}\right)}{\mathrm{d} t_{g}}=G \cdot \cos \theta-\frac{n\left(x, t_{g}\right)}{\tau(\theta)}-\frac{\mathrm{d} J\left(x, t_{g}\right)}{\mathrm{d} x}, \\
& J\left(x, t_{g}\right)=-D \frac{n\left(x, t_{g}\right)}{k_{B} T} \cdot \operatorname{grad}(U),
\end{aligned}
$$

where $n$ is the adatom density, $G$ is the incoming molecular beam flux, $J$ is the surface diffusion flux of adatoms, and $\tau, D$ and $U$ are the lifetime until incorporation, surface diffusion coefficient and chemical potential of adatoms on a facet, respectively. It is obvious that the surface lifetime, $\tau$, and the diffusion coefficient, $D$, of group III adatoms are the most important parameters that determine the growth features.

After the calculation of the adatom density, $n\left(x, t_{g}\right)$ as a function of growth time, $t_{g}$, the cross-sectional growth profile is obtained by plotting the vertical growth thickness, $T\left(x, t_{g}\right)$, which is represented by the following equation, as a function of lateral position, $x$, and growth time, $t_{g}$.

$$
T\left(x, t_{g}\right)=\int_{0}^{t_{g}} \frac{n(x, t) \cdot \Omega}{\tau \cos \theta} d t
$$

In our recent study [12], the experimentally observed growth profiles of $<-1-10>$-oriented QWRs on GaAs (001) substrates were well reproduced by computer simulation.

\subsection{Facet angle dependences of lifetime and diffusion constant}

The lifetime of group III adatoms is expected to be more strongly dependent on the step density of the growing surface than on the difference in adatom species. Thus, it is assumed here that the surface lifetimes of $\mathrm{Ga}$ adatoms and $\mathrm{Al}$ adatoms are the same, but they depend strongly on the surface slope, $\theta$, indicated as $\tau(\theta)$. In fact, the $\theta$ dependence of $\tau(\theta)$ gives the growth selectivity between the neighboring facets. 
In this study, the $\tau(\theta)$ values normalized by the lifetime on the (111)B plane, $\tau_{111 \mathrm{~B}}$, were determined from the experimentally observed growth profile on the basic one-sided mesa step given in Fig. 5, in which the side facet angle continuously changed with growth time. The growth rate ratio, $t_{\text {top }} / t_{\text {side }}$, measured from the cross-sectional image shown in Fig. 5 is plotted in Fig. 6 as a function of facet angle $\theta$. We assume that the lifetime is inversely proportional to the growth rate, and the curve indicated by the solid line in Fig. $\mathbf{6}$ gives larger $\tau(\theta) / \tau_{111 \mathrm{~B}}$. As seen in Fig. 6, a large lifetime or a high growth selectivity is obtained at an angle of $51^{\circ}$, which corresponds to the angle of the (5-12) facet with respect to the (111)B plane. This relation gives important information on the kinetic growth process on the facets having various facet angles.

As for the diffusion constant, it was assumed that $D$ is strongly dependent on the temperature, $T_{\text {sub }}$, as in the following equation $[13,14]$.

$$
D=D_{0} \exp \left(-E_{\mathrm{d}} / k T_{\text {sub }}\right)
$$

Here, $E_{\mathrm{d}}$ is the activation energy of surface adatoms diffusion.

To obtain suitable $D, E_{\mathrm{d}}$ and $\tau_{111 \mathrm{~B}}$ values for the present growth experiments, efforts were made to reproduce the experimentally obtained vertical growth rates shown in Fig. 4 quantitatively. From the repeated calculation for the theoretical fitting to the experimental data in Fig. 4, the optimum values were found to be $D_{0}=1.6 \times 10^{-6} \mathrm{~m}^{2} / \mathrm{s}, E_{\mathrm{d}}=1.2 \mathrm{eV}$ and $\tau_{111 \mathrm{~B}}$ $=1.5 \mathrm{~s}$. The results of fitting by simulation are also shown by solid lines in Fig. 4. The activation energy obtained here is much smaller than the value of $4.3 \mathrm{eV}$ for the (001) plane [12]. This indicates that the surface diffusion of adatoms on the (111)B plane is much enhanced, as compared with the case of growth on the (001) plane.

\subsection{Wire growth simulation and comparison with experiments}

Using the above fitting parameters, $\tau(\theta)$ and $D$, the growth profiles of AlGaAs layers on GaAs mesa structures were calculated for the actual growth of QWR structures. Figures 7(a) and 7(b) show the theoretical growth profiles calculated for the growth on the $<-1-12>$-oriented mesas having different widths of $W_{0}=1220 \mathrm{~nm}$ and $W_{0}=620 \mathrm{~nm}$, respectively. Growth profiles very similar to experimental ones were obtained. The facet boundaries are clearly reproduced by the simulation, and they determine the lateral width, $W$, of QWRs. The theoretically obtained $W$ values are shown by solid lines in Fig. 3. These values are in very good agreement with those experimentally obtained. From these results, it was found that the use of an initial mesa template having narrow $W_{0}$ and high $h_{0}$ is important to precisely control the lateral wire width.

Figure 8 shows the experimental and theoretical values of the facet boundary angle, $\theta_{\mathrm{b}}$, of the sample grown on a mesa with $h_{0}=250 \mathrm{~nm}$ as a function of initial mesa width, $W_{0}$. The solid curve is the theoretical curve obtained by the present simulation. Excellent agreements between experimental and theoretical values are seen in Fig. 8. These results indicate that the evolution of the facet boundary plane is the result of the difference in the atom incorporation rate between the top and side facets.

The good agreement obtained here strongly indicates that the observed complex behavior is a consequence of reaction and diffusion kinetics taking place on various facets, and that it can still be described by a phenomenological model. The computer program developed here seems to be very powerful in designing the selective growth process of QWRs. 


\section{Conclusion}

In this study, the mechanism of the cross-sectional evolution during the selective growth of $<-1-12>$-oriented GaAs quantum wires (QWRs) on (111)B-patterned substrates was studied both experimentally and theoretically. From the detailed investigation on cross sections of wires, it was found that the lateral wire width is determined by facet boundaries (FBs) within AlGaAs layers separating the growth regions between the top and side facets of mesa structures. These FBs can be planar or curved depending on the initial pattern and growth conditions. Detailed features of cross-sectional structures including the evolution of FBs could be well reproduced by computer simulation based on a phenomenological growth model considering the facet-angle-dependent lifetime of adatoms. Therefore, it is concluded that the cross sections of wires grown on (111)B-patterned substrates are kinetically controlled by the initial pattern and growth conditions, and can be well described by a computer model for advanced designs.

\section{Acknowledgement}

The work reported here is supported in part by the 21st Century COE Project at Hokkaido University on "Meme-Media Technology Approach to the R\&D of Next-Generation Information Technologies" and by a Grant-in-Aid for Young Scientists (B)-16760239 both from the Japanese Government.

\section{References}

[1] E. Kapon, D. M. Hwang and R. Bhat: Phys. Rev. Lett. 63 (1989) 430.

[2] T. Fukui, S. Ando, Y. Tokura and T. Toriyama: Appl. Phys. Lett. 58 (1991) 2018.

[3] S. Koshiba, H. Noge, H. Akiyama, T. Inoshita, Y. Nakamura, A. Shimizu, Y. Nagamune, M. Tsuchiya, H. Kano, H. Sakaki and K. Wada: Appl. Phys. Lett. 64 (1994) 363.

[4] X. L. Wang, M. Ogura and H. Matsuhata: Appl. Phys. Lett. 66 (1995) 1506.

[5] H. Hasegawa, H. Fujikura and H. Okada: MRS Bull. 24 (1999) 25.

[6] H. Hasegawa and S. Kasai: Physica E 11. (2001) 149.

[7] S. Kasai and H. Hasegawa: IEEE Electron Device Lett. 23 (2002) 446.

[8] T. Sato, I. Tamai, C. Jiang and H. Hasegawa: Inst. Phys. Conf. Ser. 170 (2002) 325.

[9] I. Tamai, T. Sato and H. Hasegawa: Physica E 21 (2004) 521.

[10] S. Yoshida, I. Tamai, T. Sato and H. Hasegawa: Jpn. J. Appl. Phys. 43 (2004) 2064.

[11] T. Sato, I. Tamai, S. Yoshida and H. Hasegawa: Appl. Surf. Sci. 234 (2004) 11.

[12] T. Sato, I. Tamai and H. Hasegawa: J. Vac. Sci. \& Technol. B 22 (2004) 2266.

[13] S. Koshiba, Y. Nakamura, M. Tsuchiya, H. Noge, H. Kano, Y. Nagamune, T. Noda and H. Sakaki: J. Appl. Phys. 76 (1994) 4138.

[14] J. M. Van Hove and P. I. Cohen: J. Cryst. Growth 81 (1987) 13. 


\section{Figures}

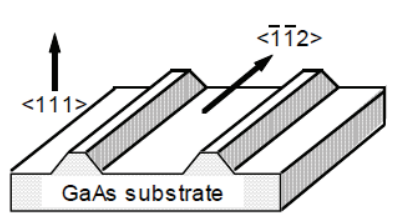

(a)

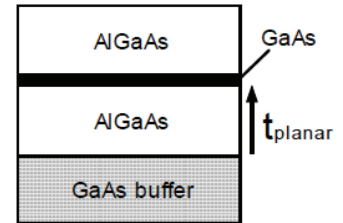

(b)

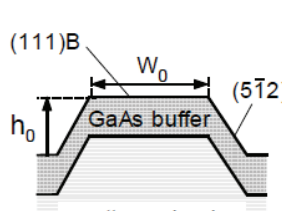

patterned sub.

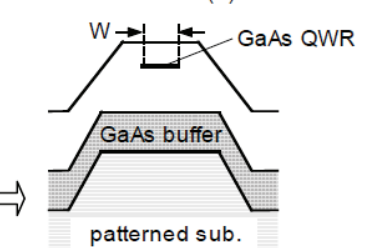

(d)
Fig. 1 Selective growth sequence used in this study. (a) Preparation of patterned substrates, (b) material supply, (c) buffer growth and (d) QWR growth.

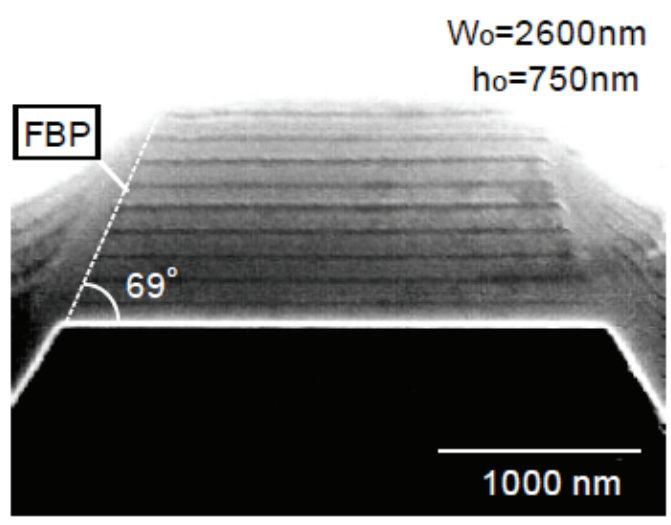

(a)

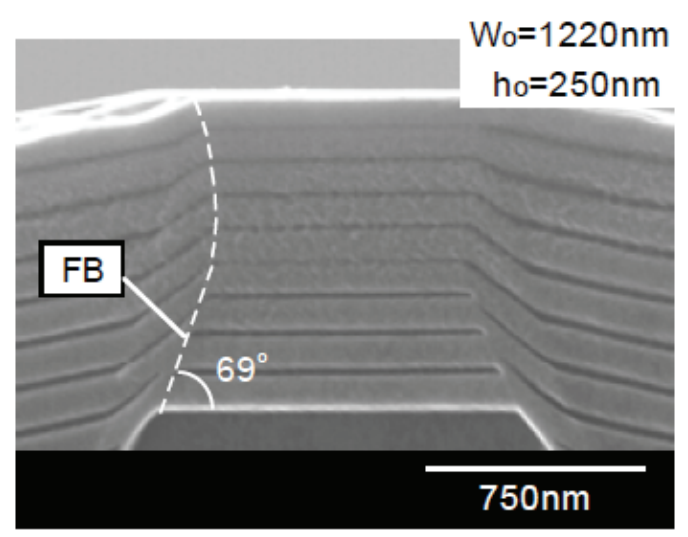

(b)

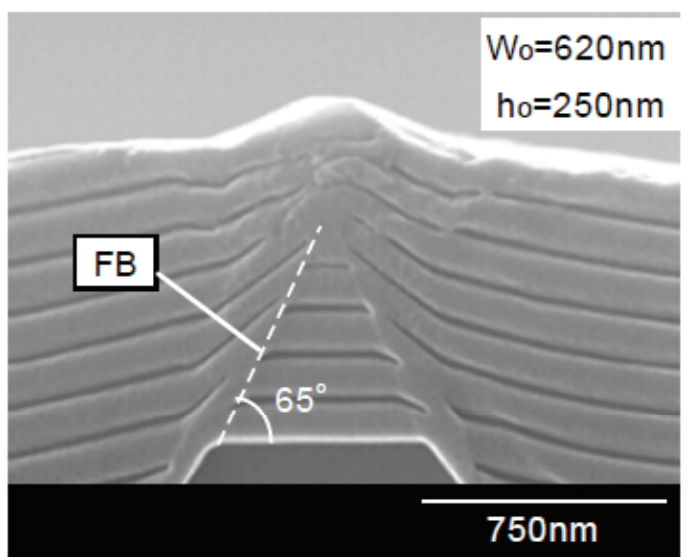

(c)

Fig. 2 Cross-sectional SEM images of samples observed after repeated wire growth on GaAs buffer mesa structures with different sizes: (a) $W_{0}=2600$ $\mathrm{nm}, h_{0}=750 \mathrm{~nm}$, (b) $W_{0}=1220 \mathrm{~nm}, h_{0}=250 \mathrm{~nm}$ and (c) $W_{0}=620 \mathrm{~nm}, h_{0}=250 \mathrm{~nm}$. The dashed lines indicate the facet boundaries (FBs). 


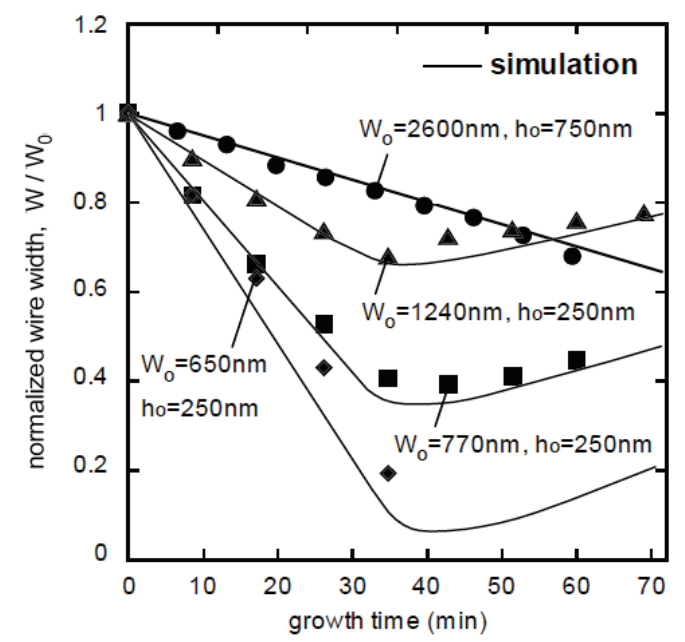

Fig. 3 Normalized wire width, $W / W_{0}$, vs growth time. Solid curves are results of computer simulation.

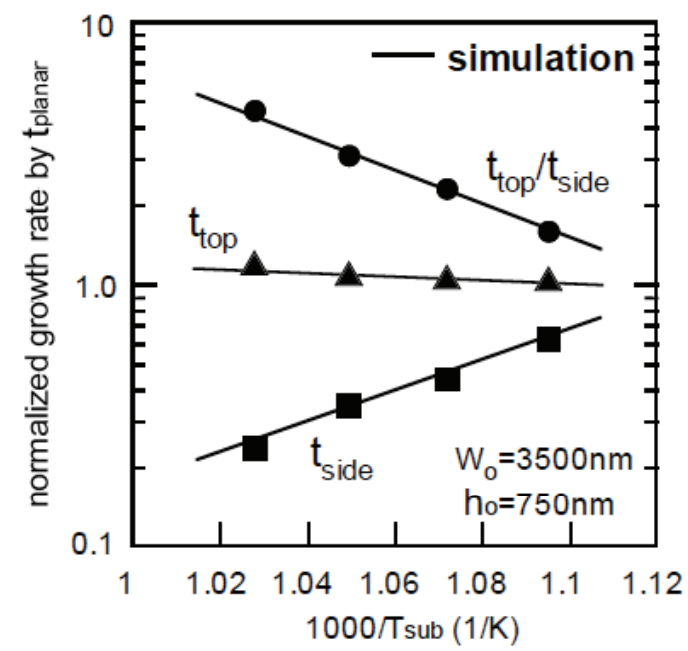

Fig. 4 Plots of normalized vertical growth rates vs $T_{\text {sub. }}$. Solid curves are results of fitting to determine fitting parameter values.

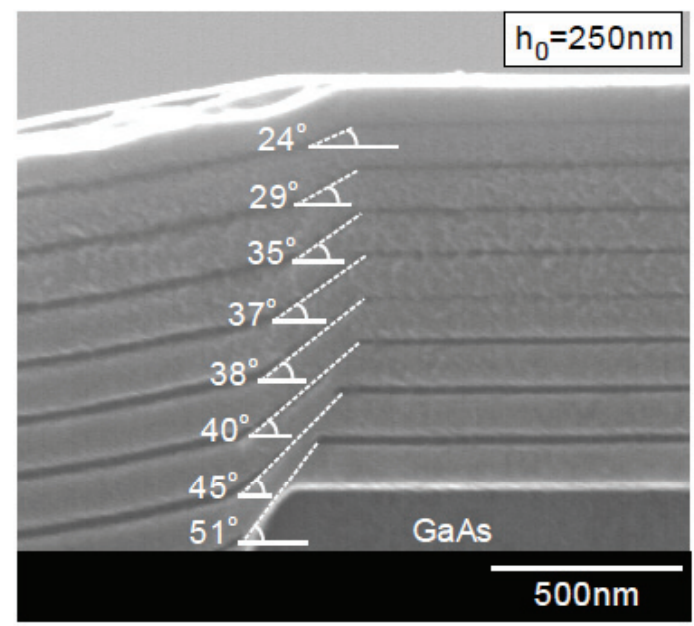

Fig. 5 Cross-sectional SEM image of sample after repeated growth of AlGaAs $(100 \mathrm{~nm}) / \mathrm{GaAs}(10 \mathrm{~nm})$ on basic one-sided mesa with $h_{0}=250 \mathrm{~nm}$.

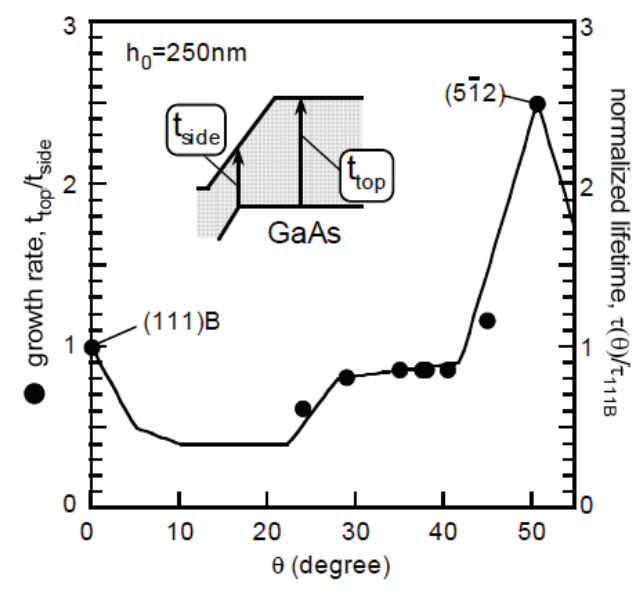

Fig. 6 Growth rate ratio, $t_{\text {top }} / t_{\text {side, }}$ vs $\theta$ determined from Fig. 5. The solid curve indicates the $\theta$ dependence of $\tau(\theta)$ normalized by the value on the (111)B plane used in the present growth simulation.

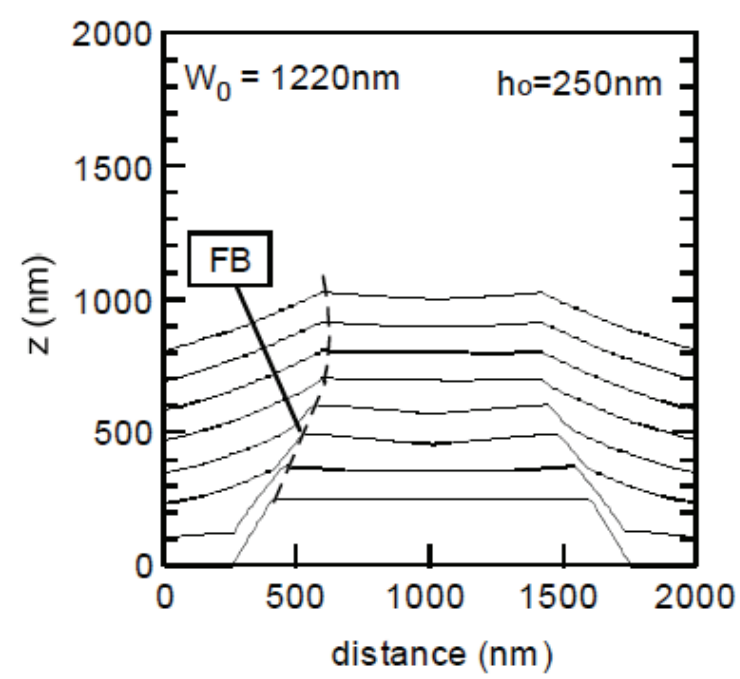

(a)

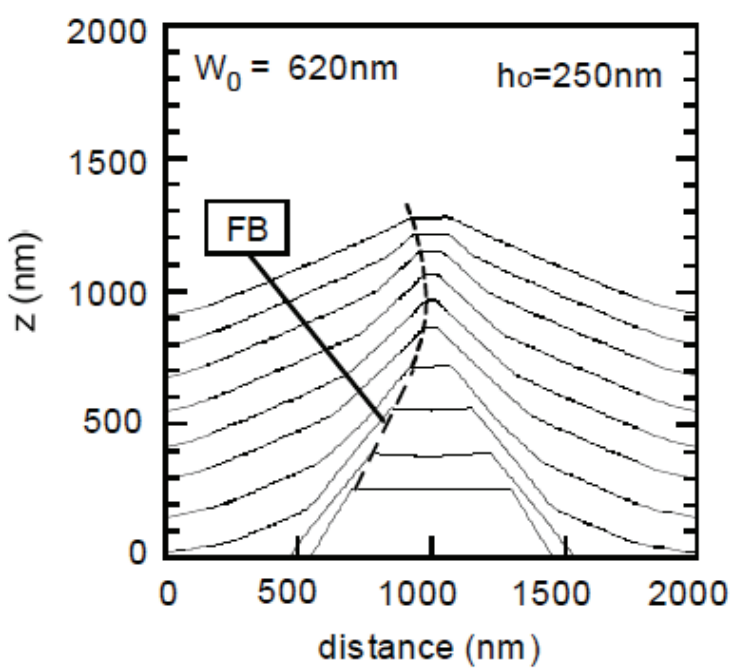

(b)

Fig. 7 Calculated growth profiles of AlGaAs layers on GaAs mesa structures: (a) $W_{0}=1220 \mathrm{~nm}$ and (b) $W_{0}=620 \mathrm{~nm}$. 


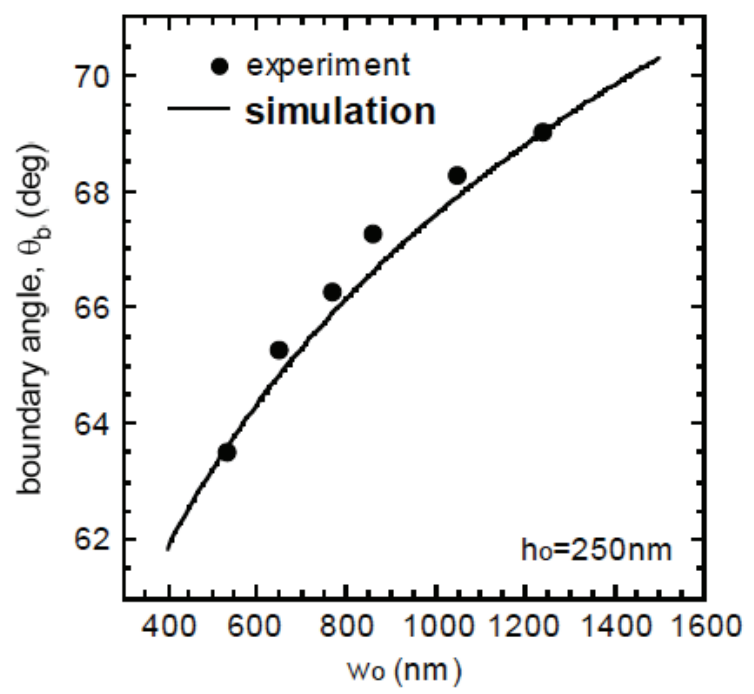

Fig. 8 Experimental and simulated values of angle of facet boundaries (FBs) plotted as functions of the initial width of the GaAs buffer mesa, $W_{0}$. 